\title{
STRATOSPHERIC VENUS OBSERVATORY
}

\author{
Cover Page
}

Mission Concept White Paper, submitted to the

Planetary Science and Astrobiology Decadal Survey 2023-2032

\author{
Eliot F. Young \\ 303-949-7944 (cell) \\ Southwest Research Institute \\ efy@boulder.swri.edu
}

Giada N. Arney < giada.n.arney@nasa.gov> NASA/GSFC

Matthew Beasley<beasley@boulder.swri.edu> SWRI

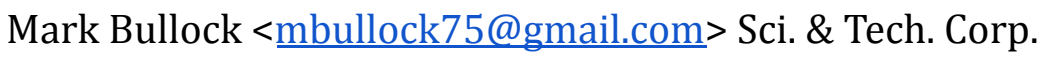

Clayton Cantrall<clca1622@colorado.edu>CU/Boulder

Nancy Chanover < nchanove@nmsu.edu $>$ New Mexico State Univ.

Jack Fox<jack.fox@comcast.net $>$ Consultant

Greg Holsclaw < Greg.Holsclaw@lasp.colorado.edu> CU/LASP

Takeshi Horinouchi <horinout@ees.hokudai.ac.jp> Hokkaido Univ.

Takeshi Imamura <t imamura@edu.k.u-tokyo.ac.jp > Univ. of Tokyo

Kandi Jessup $<$ kandilea@boulder.swri.edu $>$ SWRI

Viliam Klein<viliam@boulder.swri.edu> SWRI

Yeon Joo Lee <y.j.lee@astro.physik.tu-berlin.de> Tech. Univ. of Berlin

Sanjay Limaye < sslimaye@wisc.edu $>$ Univ. of Wisc.

Kevin McGouldrick < kevin.mcgouldrick@gmail.com> CU/LASP

William McClintock < Bill.McClintock@lasp.colorado.edu> CU/LASP

Vijay Natraj <vijay.natraj@jpl.nasa.gov> NASA/JPL

Thomas Navarro < tnavarro@epss.ucla.edu $>$ UCLA

Christopher Parkinson < cparkinson@spacescience.org > SSI

Javier Peralta <javier.peralta.calvillo@gmail.com> ISAS/JAXA

Takehiko Satoh < satoh@stp.isas.jaxa.jp> ISAS/JAXA

Constantine Tsang < con@boulder.swri.edu> SWRI

Robert A. Woodruff < rawoodruff@live.com> Consultant

Yuk Yung<yly@gps.caltech.edu> CalTech 


\section{STRATOSPHERIC VENUS OBSERVATORY}

\section{EXECUTIVE SUMMARY}

The Stratospheric Venus Observatory ( $S V O$ ) is a mission concept to study Venus's atmosphere at altitudes between the surface and $120 \mathrm{~km}$. The goal of SVO is to explore the four types of interconnected processes that control Venus's climate: circulation, atmospheric chemistry, radiative energy transfer, and cloud microphysics. A number of fundamental advances have been made in these areas since the last Decadal Survey was written, in large part due to observations made by the Venus Express (VEx) and Akatsuki missions and associated modeling efforts. SVO will surpass the spectral and spatial resolutions of Akatsuki/VEx instruments and provide a unique time-domain data set to address the suite of questions raised by those missions.

SVO observes Venus from the Earth's stratosphere. Its 3-m sparse-aperture telescope provides 10-km resolution UV images of Venus's cloud tops and 45-km resolution of Venus's middle and lower cloud decks in the $\mathrm{CO}_{2}$ windows at 1.74 and $2.3 \mu \mathrm{m}$. SVO will observe Venus in key UV bands: $198 \mathrm{~nm}$ (nitric oxide emission from 115 km [1]), 200-230 $\mathrm{nm}$ (to assess vertical mixing of $\mathrm{SO}$ and $\mathrm{SO}_{2}$ at the cloud tops [2]), $283 \mathrm{~nm}$ (exploits $\mathrm{SO}_{2}$ as a tracer at the cloud tops), and $365 \mathrm{~nm}$ (tracks the unknown UV absorber at the cloud tops). SVO will track trace gases CO, OCS, $\mathrm{H}_{2} \mathrm{O}, \mathrm{HCl}, \mathrm{HF}$, and $\mathrm{SO}_{2}$ below the cloud base in near-IR windows at 1.74 and $2.25-2.55 \mu \mathrm{m}[3,4]$ and determine cloud properties (opacity, particle sizes, $\mathrm{H}_{2} \mathrm{O} / \mathrm{H}_{2} \mathrm{SO}_{4}$ ratios and the altitude of the cloud base [5]). Observations of $\mathrm{O}_{2}{ }^{1} \Delta$ emission from $95 \mathrm{~km}$ will complement the NO emission maps to provide vertically resolved views of the subsolar to antisolar transition region. Imaging at $5 \mu \mathrm{m}$ provides cloudtop temperature maps at $\sim 66-68 \mathrm{~km}$ altitude to characterize planetary scale waves [6,7] and the time-of-day dependence of gravity waves launched from mountains on Venus [8, 9].

SVO is a timely mission for the upcoming decade. It takes advantage of NASA's new super-pressure balloon capability, which enables 100-day flights of balloon-borne telescopes that fly above $99.3 \%$ of the Earth's atmosphere. It leverages recent work on the thermal control of balloon-borne telescopes and stabilization of their focal planes. Unlike a spacecraft in orbit around Venus, SVO is recoverable and can be re-flown every 19 months during Venus inferior conjunctions. SVO corrects some of the observing deficiencies of Venus Express (cloud tracking restricted to the southern hemisphere every 24 hours) and Akatsuki (no spectral capabilities), and SVO's nearly continuous time domain coverage is well-suited to provide data for assimilation into GCMs that have been developed in response to Venus Express and Akatsuki observations (e.g., [10]).

\section{MISSION AND INSTRUMENT OVERVIEW}

NASA's balloon program regularly flies payloads weighing several tons to altitudes of 33 $40 \mathrm{~km}$, above $99.3-99.7 \%$ of the Earth's atmosphere, respectively. The inner Fried para- 
meter is at least $4 \mathrm{~m}$ at those altitudes [11]: balloon-borne telescopes with 4-m apertures can produce diffraction-limited images, even at UV wavelengths where ground-based adaptive optics perform poorly. Balloon-borne telescopes have nearly complete access to the spectrum from $0.2-5 \mu \mathrm{m}$, except for a drop-out between $230-280 \mathrm{~nm}$ and partial obscuration at 4.3 and $4.6 \mu \mathrm{m}$ due to $\mathrm{CO}_{2}$ and $\mathrm{CO}$ (Fig. 1).

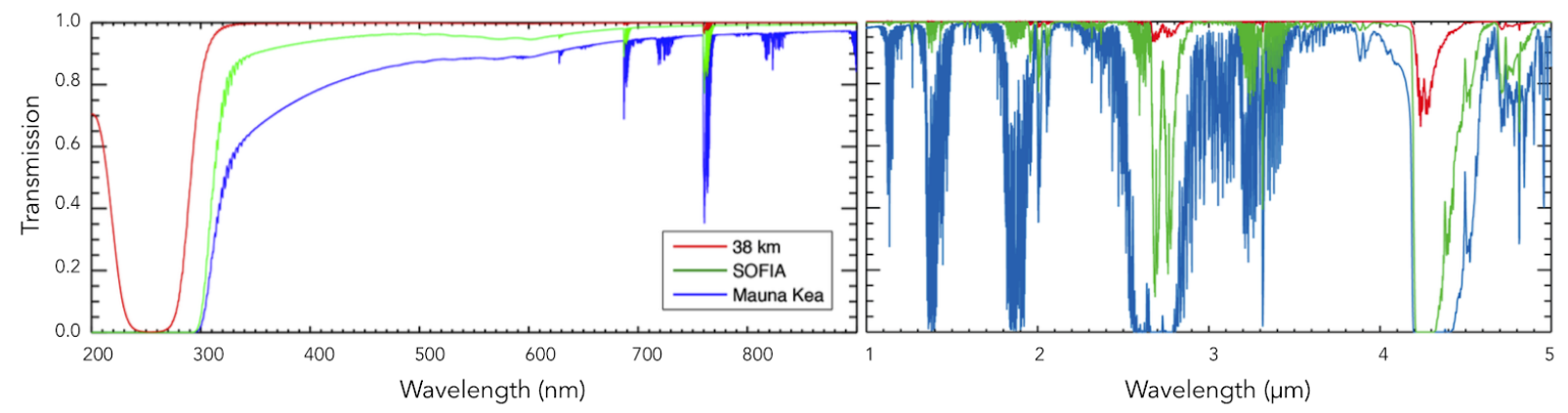

Fig. 1 From [12]: MODTRAN simulations of the telluric transmission between $200 \mathrm{~nm}$ to $5 \mu \mathrm{m}$ from $38 \mathrm{~km}$ (red), showing stratospheric access to UV and IR wavelengths.

Telescope: For SVO, we propose a 3-m aperture telescope with separate UV and IR cameras to obtain spectral image cubes of Venus over key wavelength ranges. Because a monolithic 3-m mirror - even aggressively lightweighted - would be prohibitively heavy, SVO uses a Golay3 design: three 1-m mirrors with their centers separated by $2 \mathrm{~m}$. The resulting PSF has a central core that is narrower than that of a monolithic 3-m mirror, surrounded by six lobes that peak at $30 \%$ of the height of the central core (Fig. 2). This PSF would not be ideal for imaging exoplanets around stars, but it works well for the relatively low-contrast problem of tracking clouds or mapping trace gas distributions on Venus.

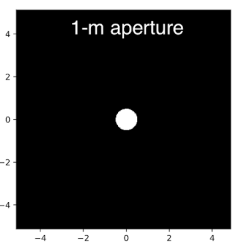

Meters

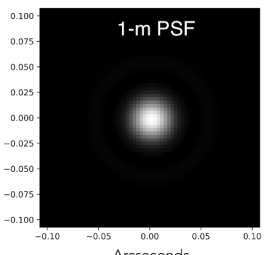

Arcseconds

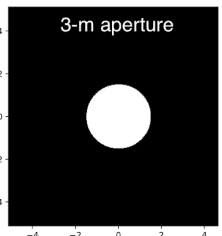

Meters

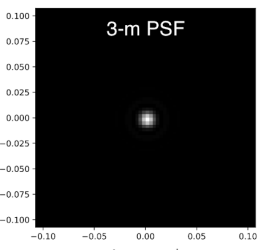

Arcseconds

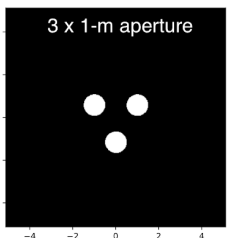

Meters

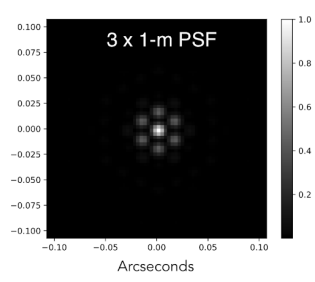

Fig. 2 The top row shows three apertures, consisting of a 1-m mirror, a 3-m mirror and a sparse aperture (3 separate 1-m mirrors). The bottom row shows the Point Spread Functions that each aperture would produce, normalized to the peak of each PSF. The central lobe of the $3 x$ $1-m$ aperture is slightly narrower than the 3-m PSF; the six side-lobes peaks are $30 \%$ as high as the central peak.

Image Quality: Balloon-borne telescopes suffer from thermal gradients and image motion. SVO leverages recent work from NASA's THAI-SPICE and SuperBIT balloon missions: THAI-SPICE investigated passive thermal shielding as a means to drastically reduce thermal 
gradients across balloon-borne telescopes. For SVO, each 1-m mirror has an articulated sugar-scoop baffle to act as a sunshield. Several balloon missions (e.g., HySICS, BOPPS, SuperBIT) have demonstrated coarse pointing systems that stabilize a telescope at the few arc-second level. SVO, like THAI-SPICE, uses an orthogonal transfer CCD (OTCCD) camera in UV-visible wavelengths to perform fine stabilization: on-chip tip-tilt corrections at $\mathrm{kHz}$ rates to stabilize the focal plane at the 0.02 " level (rms). In wavelengths from $0.8-5 \mu \mathrm{m}$, SVO can either use an HgCdTe array that is bump-bonded to an OTCCD to provide tip-tilt correction or SAPHIRA HgCdTe arrays (from Leonardo S.p.A.) with avalanche read-out electronics. The latter allows very fast frame rates with negligible read noise, but requires co-registration of frames in software and is currently limited to $\lambda=0.8-2.5 \mu \mathrm{m}$.

Instrumentation: SVO has two instruments, UVCam and IRCam, operating simultaneously via a dichroic with a pivot at $0.7 \mu \mathrm{m}$. UVCam obtains spectral image cubes from $200-600$ $\mathrm{nm}(R=100)$, IRCam from 0.8 to $5 \mu \mathrm{m}(R=500$ at $2.25-2.55 \mu \mathrm{m}$, plus narrow fixed filters for $\mathrm{SO}_{2}$ characterization). SVO also has two guide cameras (wide-field and narrow-field), similar to those on the THAI-SPICE mission. The narrow-field guide camera provides $0.02 "$ (rms) pointing error updates at $100 \mathrm{~Hz}$. The 0.02 " pointing requirement is driven by the 0.024 " diffraction limit of a 3-m aperture at $\lambda=283 \mathrm{~nm}$. UVCam and IRCam use fixed and tunable filters (e.g., Acousto-Optic Tunable Filters as described in [13]) to identify trace gases (CO, OCS, $\mathrm{H}_{2} \mathrm{O}, \mathrm{HCl}, \mathrm{HF}$, and $\mathrm{SO}_{2}$ below the cloud base, and the $\mathrm{SO} / \mathrm{SO}_{2}$ ratio at the cloud tops), constrain the composition of the unknown ultraviolet absorber, detect airglow from $\mathrm{NO}$ and $\mathrm{O}_{2}$ and to identify cloud properties (opacity, particle sizes, $\mathrm{H}_{2} \mathrm{O} / \mathrm{H}_{2} \mathrm{SO}_{4}$ ratios and cloud base altitudes).

Mission Opportunities: SVO observes Venus during the 100-day periods around Venus's inferior conjunctions, when its angular diameter ranges from $\sim 32$ " to $\sim 60$ " and the dayside crescent is $38 \%$ of the disk or less. These circumstances let SVO observe several phenomena on Venus's nightside: cloud-tracking in the middle and lower cloud decks (48-55 km altitude), trace gases $\left(0-48 \mathrm{~km}\right.$ ) and airglow (from NO and $\mathrm{O}_{2}$ ). On the dayside, SVO observes the cloud tops (10-km resolution), UV spectra diagnostic of the unknown UV absorber, and the $\mathrm{SO} / \mathrm{SO}_{2}$ ratio.

NASA's 100-day super-pressure balloon flights will launch from McMurdo Bay or Wanaka, NZ, since long flights in the northern hemisphere are precluded from flying over Russia. This means that the inferior conjunctions on 25-OCT-2026 and 06-JAN-2030 are preferred: Venus's southern declination during those events allows a balloon-borne telescope to observe Venus for 16 out of 24 hours each day from Wanaka latitudes or 23 out of 24 hours from McMurdo (Fig. 3). Daytime observing is possible at all wavelengths, but the sky background is significant at UV wavelengths (albeit $\sim 100 \mathrm{x}$ less at balloon altitudes than at the surface). Short exposures with co-adds are required to avoid daytime saturation in UV wavelengths. Daytime observations of Venus in IR bands (0.8 to $5 \mu \mathrm{m})$ are routinely 


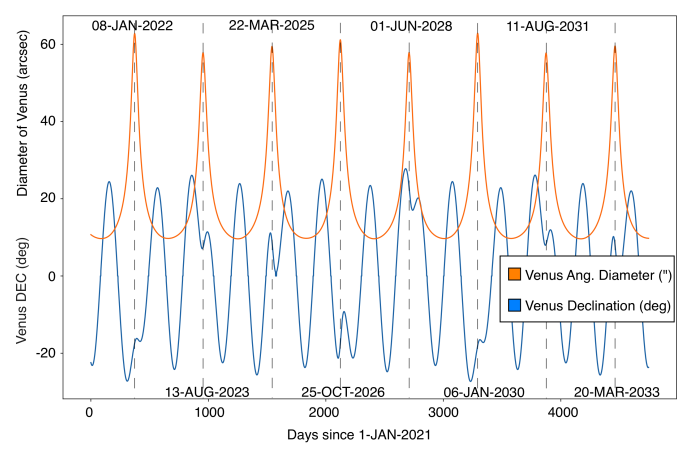

Table 1. Goal II (Understanding Atmospheric Dynamics and Composition) from The VEXAG document Goals, Objectives and Investigations (2019).

\begin{tabular}{|c|c|}
\hline Objective & Investigation \\
\hline \multirow{3}{*}{$\begin{array}{l}\text { A. What } \\
\text { processes drive } \\
\text { the global } \\
\text { atmospheric } \\
\text { dynamics of } \\
\text { Venus? }\end{array}$} & $\begin{array}{l}\text { DD. Deep Dynamics (1). Characterize the dynamics of the } \\
\text { lower atmosphere (below about } 75 \mathrm{~km} \text { ) of Venus, including: } \\
\text { retrograde zonal super-rotation, meridional circulation, } \\
\text { radiative balances, mountain waves, and transfer of angular } \\
\text { momentum. }\end{array}$ \\
\hline & $\begin{array}{l}\text { UD. Upper Dynamics (1). In the upper atmosphere and } \\
\text { thermosphere of Venus, characterize global dynamics and } \\
\text { interactions between space weather and the ionosphere and } \\
\text { magnetosphere. }\end{array}$ \\
\hline & $\begin{array}{l}\text { MP. Mesoscale Processes (2). Determine the role of } \\
\text { mesoscale dynamics in redistributing energy and momentum } \\
\text { throughout the atmosphere of Venus. }\end{array}$ \\
\hline \multirow{5}{*}{\begin{tabular}{|c|} 
\\
B. What \\
processes \\
determine the \\
baseline and \\
variations in \\
Venus \\
atmospheric \\
composition and \\
global and local \\
radiative \\
balance? \\
\end{tabular}} & $\begin{array}{l}\text { RB. Radiative Balance (1). Characterize atmospheric } \\
\text { radiative balance and how radiative transport drives } \\
\text { atmospheric dynamics on Venus. }\end{array}$ \\
\hline & $\begin{array}{l}\text { IN. Interactions (1). Characterize the nature of the physical, } \\
\text { chemical, and possible biological interactions among the } \\
\text { constituents of the Venus atmosphere. }\end{array}$ \\
\hline & $\begin{array}{l}\text { AE. Aerosols (2). Determine the physical characteristics and } \\
\text { chemical compositions of aerosols in Venus atmosphere as } \\
\text { they vary with elevation, including discrimination of aerosol } \\
\text { types/components. }\end{array}$ \\
\hline & $\begin{array}{l}\text { UA. Unknown Absorber (2). Characterize the unknown short- } \\
\text { wavelength absorber in the upper atmosphere of Venus and } \\
\text { its influence on local and global processes. }\end{array}$ \\
\hline & $\begin{array}{l}\text { OG. Outgassing (3). Determine the products of volcanic } \\
\text { outgassing on Venus and their effects on atmospheric } \\
\text { composition. }\end{array}$ \\
\hline
\end{tabular}

conducted from ground-based observatories and will be trivial from the stratosphere.

Fig. 3 Venus's angular diameter (orange) gets as large as 62" during inferior conjunctions every 19 months. Venus's declination varies roughly from +20 to -20 degrees. Southern declinations are preferred, due to NASA's balloon launch sites in the southern hemisphere.

\section{SCIENCE JUSTIFICATION AND RELEVANCE TO KEY QUESTIONS}

SVO will monitor and measure most of the processes that have been identified in VEXAG Goal II: Understand atmospheric dynamics and composition on Venus in the most recent VEXAG (Venus Exploration Analysis Group) GOI document (Goals, Objectives and Investigations) [14]. Table 1 lists eight key investigations (Deep Dynamics, etc.), and SVO addresses all of them with the possible exceptions of $U D$ and $O G$. We now list SVO's investigations, broken down into four categories (Dynamics, Atmospheric Chemistry, Radiative Balance and Cloud Microphysics) and referenced to the VEXAG investigations in Table 1.

Dynamics: SVO will track atmospheric motion from the surface to $120 \mathrm{~km}$ with four types of observations: monitoring trace gas distributions from the surface to the cloud base (observing $\mathrm{H}_{2} \mathrm{O}, \mathrm{CO}, \mathrm{OCS}, \mathrm{HCl}, \mathrm{HF}$, and $\mathrm{SO}_{2}$ at 1.71-1.74 $\mu \mathrm{m}$ and 2.25-2.5 $\mu \mathrm{m}$ with expected spatial resolutions of $\sim 50-100 \mathrm{~km}$ and velocity resolutions of 7-15 m/s); tracking clouds on Venus's nightside in the lower and middle cloud decks (observing cloud silhouettes at 1.74 and $2.3 \mu \mathrm{m}, 48-55 \mathrm{~km}$ altitude, with spatial resolutions of $\sim 42-51 \mathrm{~km}$ and velocity resolution of $1 \mathrm{~m} / \mathrm{s}$ ); tracking dayside clouds at or near the cloud tops (at 200-230 nm to assess vertical mixing, at $283 \mathrm{~nm}$ to track $\mathrm{SO}_{2}$, at $365 \mathrm{~nm}$ to track the unknown absorber all with a spatial resolution of $10 \mathrm{~km}$ and velocity resolution of $0.25 \mathrm{~m} / \mathrm{s}$, plus imaging at 5 $\mu \mathrm{m}$ to generate cloudtop thermal maps at $\sim 66-68 \mathrm{~km}$ altitude with $\sim 112 \mathrm{~km}$ spatial 
resolution); and monitoring airglow from $\mathrm{O}_{2}{ }^{1} \Delta$ and NO (at altitudes of 95 and $115 \mathrm{~km}$ with expected spatial resolution of $29 \mathrm{~km}$ and velocity resolutions of 3-5 m/s).

Significance: (1) GCM models suggest that Venus has Hadley cell-like circulation (e.g. [15]), but complete Hadley cells have never been observed. Cloud-tracking studies at $70 \mathrm{~km}$ show that meridional motion at the cloudtops is poleward, as expected for the top of equatorial-high latitude Hadley cells. The return branch beneath the clouds has never been observed. SVO will provide long stares at advecting trace gas distributions to obtain rough wind maps between 0-48 km. SVO also obtains nightside cloud opacities to deduce "wet" or "dry" regions and to constrain areas of upwelling and downwelling. (2) Recent explanations for Venus's super-rotating atmosphere [Horinouchi et al., 2020] predict patterns of mean meridional and mean zonal cloud motion. SVO will resolve horizontal motion in the lower/middle cloud decks and at the cloud tops and help confirm these predictions. (3) circulation models can now simulate mountain waves [9] and planetary scale waves, both of which are observables at the cloud tops for SVO's $5 \mu \mathrm{m}$ filter and represent an important synergy between observations and modeling efforts. (4) Altitudes from $\sim 90-120 \mathrm{~km}$ represent a transition region, where subsolar-to- antisolar (SS_AS) winds dominate the background retrograde zonal flow. Observations of $\mathrm{NO}$ and $\mathrm{O}_{2}$ emission will track the convergent flow at two levels in the transition region and help assess the transfer of angular momentum through that region. These combined observations address the VEXAG investigations $D D, M P$ and $U A$.

Atmospheric Chemistry: SVO will map trace gas distributions through spectral windows in Venus's $\mathrm{CO}_{2}$ atmosphere (Fig. 4). [17] used CO from Venus Express/VIRTIS spectra as a dynamical tracer below the cloud base, but with significant spatial binning to improve their SNR. SVO is further from Venus than Venus Express was, but its larger aperture and much longer integration times produce maps with SNRs better than VIRTIS by an order of magnitude and spatial resolutions of $\sim 45 \mathrm{~km}$, similar to VIRTIS. SVO will retrieve $\mathbf{S O}$ and $\mathbf{S O}_{2}$ at the cloud tops from $200-230 \mathrm{~nm}$ spectra; multi-level $\mathbf{H}_{2} \mathbf{O}$ distributions from three spectral windows $(1.18,1.74 \& 2.4 \mu \mathrm{m})$ to track advection below the cloud base; $\mathbf{S O}_{2}$ and CO below the cloud base $(2.3,2.53 \mu \mathrm{m})$; and ratios of OCS and CO from 2-11 bar.

Significance: (1) $\mathrm{SO}$ and $\mathrm{SO}_{2}$ have different (but short) photolysis lifetimes at the cloud tops. The $\mathrm{SO} / \mathrm{SO}_{2}$ ratio is therefore diagnostic of vertical mixing just below the cloud tops and constrains convective activity, especially given SVO's spatial resolution of $10 \mathrm{~km}$, commensurate with the size of convection cells [18]. (2) $\mathrm{H}_{2} \mathrm{O}$ and $\mathrm{SO}_{2}$ distributions below the cloud base - combined with dynamics inferred from cloud tracking and cloud opacities will constrain competing models of cloud formation [19, 20]. (3) CO is thought to be a dynamical tracer of Hadley cell circulation [21]: SVO tracking of CO will help identify the lower return branch of the Hadley cell. (4) Descending CO is thought to be converted to OCS $[22,23]$ : SVO will provide high SNR maps of both species, characterizing their chemical and spatial relationship. (5) $\mathrm{H}_{2} \mathrm{O}$ can serve as a dynamical tracer at three different levels from 
the surface to the cloud base (Fig. 4). These combined observations address the VEXAG investigations IN and $A E$.

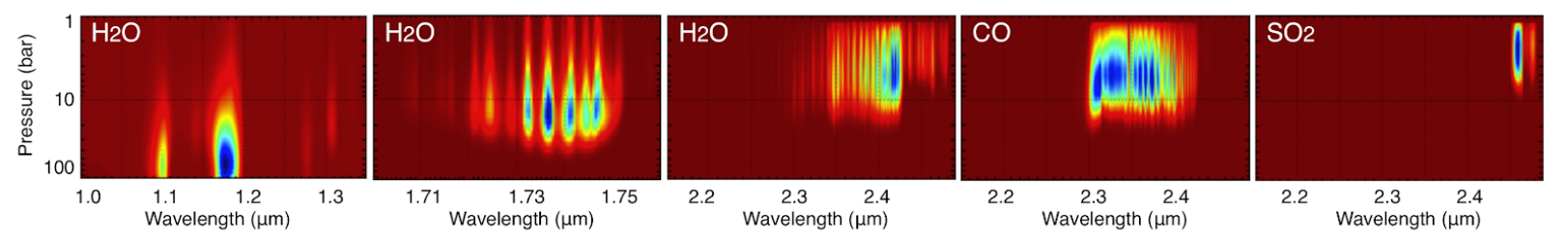

Fig. 4 Jacobians showing where (in altitude or pressure) and at what wavelengths we have spectral sensitivities to trace gases $\mathrm{H}_{2} \mathrm{O}, \mathrm{CO}$ and $\mathrm{SO}_{2}$ - similar plots exist for OCS, $\mathrm{HF}$ and $\mathrm{HCl}$ [Tsang et al. 2007].

Radiative Balance \& Cloud Microphysics: SVO maps Venus's radiative sources and sinks in several ways. $5 \mu \mathrm{m}$ images map Venus's temperature at altitudes near $66 \mathrm{~km}$. Maps of the cloud tops at $365 \mathrm{~nm}$ (at 10-km resolution) show where the unknown UV absorber actually does its absorbing, while mapping motions of convection cells at the same time. SVO maps cloud optical depths in the lower and middle cloud decks, plus the altitude of the upper cloud deck (via images at 1.65 and $2.02 \mu \mathrm{m}$, which measure overlaying $\mathrm{CO}_{2}$ columns). These cloud optical depths, combined with abundance maps of Venus's greenhouse gases $\left(\mathrm{H}_{2} \mathrm{O}, \mathrm{SO}_{2} \& \mathrm{CO}\right)$ let us model vertical heat transfer via radiative transfer codes. Combined IR images at 1.74 and $2.3 \mu \mathrm{m}$ are diagnostic of cloud particle sizes.

Significance: (1) SVO observes cloud opacities and greenhouse gas distributions at 45-km resolution, both of which serve as inputs to GCMs. SVO also provides simultaneous comparisons to GCM outputs in the form of observed cloud motions with $1 \mathrm{~m} / \mathrm{s}$ resolution. (2) At the cloud tops, SVO provides simultaneous GCM inputs (maps of the unknown UV absorber, absorbing roughly $50 \%$ of incident sunlight) and comparisons to GCM outputs (cloud motions at the cloud tops, at 10-km resolution). (3) SVO's $5 \mu$ m images allow further synergies with GCMS: they show stationary features due to orographically launched mountain waves and, with Fourier analysis, the presence of planetary scale waves. These combined observations address the VEXAG investigations $R B, A E$ and $U A$.

\section{SUPPORT FOR BALLOON MISSIONS AND NEW TECHNOLOGIES}

We encourage the Planetary Science and Astrobiology Decadal Survey to advocate for SVO in two specific areas. First, NASA's Planetary Science Division is unique among the four divisions of SMD in having no funding avenues to support balloon-borne research. Astrophysics' APRA program supports the majority of NASA's (non-planetary) balloon payloads. Furthermore, the new Astrophysics Pioneers program supports small satellites and major balloon payloads at the \$20M level, about 5-10 times higher than typical APRA awards. The need for balloon platforms to address planetary science goals has been recognized for years: the current Decadal Survey (Visions and Voyages) [24] includes text in support of planetary balloon missions, and [25] identified roughly $20 \%$ of the key science questions in Visions and Voyages as candidates for balloon-borne investigations. We 
encourage the Decadal Survey to advocate for changes to NASA/PSD AOs, specifically, to allow balloon missions to compete in research programs (like SIMPLEx) and to consider the creation of a Planetary Pioneers program. Second, there are promising technological areas that need development. SVO uses four in particular: narrow-band UV filters between 200-230 nm, phased mirror segments in a sparse aperture telescope, tunable filters with narrow bandpasses in UV and solid-state motion-compensation arrays. NASA's Earth Science Division Technology Office (https://esto.nasa.gov) has a strong suite of coordinated research programs to advance technologies. It is our perception that the PSD analog (PESTO) is much more limited than ESTO. We encourage the Decadal Survey to suggest that NASA's Planetary Science Division emulate the ESTO programs in range (spanning broad, low-TRL projects to satellites) and scope (funding levels).

[1] Gérard J. C. et al. (2009) Concurrent observations of the ultraviolet nitric oxide and infrared 02 nightglow emissions with Venus Express. JGR: Planets, 114.

[2] Jessup, K. L.et al., (2015) Coordinated Hubble Space Telescope and Venus Express Observations of Venus' upper cloud deck. Icarus. 258, 309-336.

[3] Arney, G. et al. (2014) Spatially resolved measurements of $\mathrm{H} 2 \mathrm{O}, \mathrm{HCl}, \mathrm{CO}, \mathrm{OCS}, \mathrm{SO} 2$, cloud opacity, and acid concentration in the Venus near-infrared spectral windows. JGR: Planets.

[4] Pollack J. B. et al. (1993) Near infrared light from Venus' nightside: A spectroscopic analysis. Icarus, 103: 1-42.

[5] Barstow, J. K.et al. (2012) Models of the global cloud structure on Venus derived from Venus Express observations. Icarus. 217, 542-560.

[6] Fukuhara, T. et al. (2017) Large stationary gravity wave in the atmosphere of Venus. Nature Geoscience. 10, 85-89.

[7] Imamura, T. et al. (2019) Planetary-scale waves on Venus found in the cloud-top temperature. EPSC-DPS Joint Meeting 2019.

[8] Kouyama, T. et al. (2017) Topographical and Local Time Dependence of Large Stationary Gravity Waves Observed at the Cloud Top of Venus. GRL. 44, 12,098-12,105.

[9] Navarro, T., Schubert, G., Lebonnois, S. (2018) Atmospheric mountain wave generation on Venus and its influence on the solid planet's rotation rate. Nature Geoscience. 11, 487-491.

[10] Ando H. et al. (2020) Venusian cloud distribution simulated by a general circulation model. JGR: Planets, 125: e2019JE006208.

[11] Ford, H. C. et al. (2002) Artemis: a stratospheric planet finder, Adv. Space Res., 30, 1283-1288.

[12] Chanover, N. J. et al. (2016) Findings Report for the Gondola for High Altitude Planetary Science/Science Instrument Definition Team.

[13] Chanover, N. J. et al. (2015) A balloon-borne Acousto-Optic Tunable Filter imaging camera for planetary science investigations. DOI: 10.1109/AERO.2015.7119118.

[14] VEXAG. (2019) Goals, Objective, and Investigations for Venus Exploration.
https://www.lpi.usra.edu/vexag/reports/VEXAG_Venus_GOI_ Current.pdf

[15] Lebonnois S. et al. (2010) Superrotation of Venus' atmosphere analyzed with a full general circulation model. JGR (Planets), 115: 6006.

[16] Horinouchi, T. and 16 colleagues (2020) How waves and turbulence maintain the super-rotation of Venus' atmosphere. Science 368, 405-409.

[17] Tsang, C. C. C. and 9 colleagues (2008) Tropospheric carbon monoxide concentrations and variability on Venus from Venus Express/VIRTIS-M observations. Journal of Geophysical Research (Planets) 113.

[18] Lefèvre, M., Lebonnois, S., Spiga, A. (2018) Three-Dimensional Turbulence-Resolving Modeling of the Venusian Cloud Layer and Induced Gravity Waves: Inclusion of Complete Radiative Transfer and Wind Shear. JGR (Planets) 123, 2773-2789.

[19] Shao W. D. et al. (2020) Revisiting the Sulfur-Water Chemical System in the Middle Atmosphere of Venus. JGR: Planets: e2019JE006195.

[20] Parkinson C. D. et al. (2015) Distribution of sulphuric acid aerosols in the clouds and upper haze of Venus using Venus Express VAST and VeRa temperature profiles. P\&SS, 113,: 205.

[21] Tsang, C. C. C. et al. (2008) A correlated-k model of radiative transfer in the near-infrared windows of Venus. JSQRT 109, 1118-1135.

[22] Krasnopolsky, V. A. (2007) Chemical kinetic model for the lower atmosphere of Venus. Icarus 191, 25-37.

[23] Yung, Y., Liang, M. 2008. Modeling the Distribution of CO in the Atmosphere of Venus. AAS/Division for Planetary Sciences Meeting Abstracts \#40.

[24] National Academies of Sciences, Engineering, and Medicine, 2018. Visions into Voyages for Planetary Science in the Decade 2013-2022: A Midterm Review. DOI: 10.17226/25186

[25] Dankanich J. W. et al. (2016) Planetary Balloon-Based Science Platform Evaluation and Program Implementation. https://ntrs.nasa.gov/citations/2016000 